\title{
Computably Categorical Fields via Fermat's Last Theorem
}

\author{
Russell Miller \\ Department of Mathematics \\ Queens College - C.U.N.Y. \\ 65-30 Kissena Blvd. \\ Flushing, New York 11367 U.S.A. \\ Ph.D. Programs in Mathematics \& Computer Science \\ C.U.N.Y. Graduate Center \\ 365 Fifth Avenue \\ New York, New York 10016 U.S.A. \\ Russell.Miller@qc.cuny.edu \\ qcpages.qc.cuny.edu/ rmiller \\ Hans Schoutens \\ Department of Mathematics \\ New York City College of Technology \\ 300 Jay Street \\ Brooklyn, New York 11201 U.S.A. \\ Ph.D. Program in Mathematics \\ C.U.N.Y. Graduate Center \\ 365 Fifth Avenue \\ New York, New York 10016 U.S.A. \\ hschoutens@citytech.cuny.edu \\ websupport1.citytech. cuny.edu/faculty/hschoutens/
}

\begin{abstract}
We construct a computable, computably categorical field of infinite transcendence degree over the rational numbers, using the Fermat polynomials and assorted results from algebraic geometry. We also show that this field has an intrinsically computable (infinite) transcendence basis.
\end{abstract}

Keywords: Computability, Computable Categoricity, Fermat's Last Theorem, Fields, Transcendence

\section{Introduction}

In computable model theory, we investigate the extent to which various model-theoretic constructions can be performed effectively. For instance, given two structures, model theorists naturally wish to consider isomorphisms between them. In computable model theory, we break this down into two problems. First we pose the Isomorphism Problem, in which we ask how difficult it is to determine in general whether two given structures are isomorphic to each other at all. If they are indeed isomorphic, then we ask about the difficulty of actually computing an isomorphism between them. This latter question involves the notion of computable categoricity. When asking these questions, we usually assume that the structures themselves are computable, meaning that we can compute the functions and relations on them. If they are in fact isomorphic, then it is reasonable to ask whether there exists a computable isomorphism between them.

Fields were the first mathematical structures for which the notion of computable categoricity arose. The isomorphism problem for fields is addressed by Calvert in [2]. Long before that, though, in [8], Frohlich and Shepherdson had begun to consider the second question, by giving an example of (in their terminology; see their Corollary 5.51) two isomorphic, explicitly presented fields with no explicit isomorphism between them. This idea eventually grew into the following definition. 
Definition 1.1. A computable structure $\mathscr{A}$ is computably categorical if for every computable structure $\mathscr{B}$ isomorphic to $\mathscr{A}$, there exists a computable isomorphism from $\mathscr{A}$ onto $\mathscr{B}$.

From the point of view of our second question, such a structure is about as nice as it could be: no matter which two computable copies of $\mathscr{A}$ we choose, there must exist a computable isomorphism between them. (An even nicer version, called uniform computable categoricity and examined in [4], requires not only that a computable isomorphism exist, but that we should be able to figure out a program for computing it, just given the ability to compute the structures $\mathscr{A}$ and $\mathscr{B}$.) If the structure is not computably categorical, we may ask how strong an oracle is required to compute isomorphisms between two computable copies; for a consideration of this question for algebraic fields, for example, see [27].

Much research has been devoted to characterizing the computably categorical models of various theories, including work by Dzgoev, Goncharov, Khisamiev, Lempp, McCoy, Miller, Remmel, and Solomon. Some results are readily stated: we know that a computable linear order is computably categorical if and only if it has only finitely many pairs of consecutive elements, for example, and that a computable Boolean algebra is computably categorical if and only if it has finitely many atoms. On the other hand, the known structural characterization of computably categorical trees requires a description by recursion on the heights of finite trees. The question has been studied for a number of other theories as well, and results along these lines may be found in [9], [10], [11], [12], [13], [19], [21], [24], [31], and [32].

However, the original problem of computable categoricity for fields has defied all attempts at structural characterization. The most obvious conjecture would be that the transcendence degree of a field over its prime subfield should determine computable categoricity. For algebraically closed fields, this is indeed the case, as shown by Ershov in [5]: an ACF is computably categorical if and only if it has finite transcendence degree over its prime subfield. However, in the same work, Ershov built a field, algebraic over its prime subfield but not algebraically closed, which was not computably categorical, and a number of further results for algebraic fields have been developed in [28] by Shlapentokh and one of us, who then extended them in [17] jointly with Hirschfeldt and Kramer. In this paper we refute the converse as well, by constructing a computably categorical field of infinite transcendence degree over the rationals $\mathbb{Q}$. Thus, neither implication in the original conjecture actually holds.

The counterexample $F$ we build is readily described. It begins with an infinite, purely transcendental extension $\mathbb{Q}\left(x_{0}, x_{1}, \ldots\right)$ of $\mathbb{Q}$. Then, for each $i$, we adjoin an element $y_{i}$ such that $\left(x_{i}, y_{i}\right)$ is a solution of the Fermat curve $D_{p_{i}}$ defined by $X_{i}^{p_{i}}+Y_{i}^{p_{i}}=1$, for an odd prime $p_{i}$. Thus each $y_{i}$ is algebraic over $x_{i}$, but the infinite set $\left\{x_{i}: i \in \omega\right\}$ is still algebraically independent. Fermat's Last Theorem shows that each $D_{p_{i}}$ has only the trivial solutions $(0,1)$ and $(1,0)$ in $\mathbb{Q}$, and the heart of our proof is a demonstration that there exists a computable sequence $p_{0}<p_{1}<\cdots$ such that in $F$, every nontrivial solution of the equation of $D_{p_{i}}$ generates the same subfield, namely $\mathbb{Q}\left(x_{i}, y_{i}\right)$. Therefore, mapping $x_{i}$ and $y_{i}$ to any nontrivial solution of $D_{p_{i}}$ in a field $\tilde{F}$ isomorphic to $F$ will define an isomorphism. The algebraic geometry required is developed in Section 2, and the sequence $\left\langle p_{i}\right\rangle$ is chosen and the field presented in Section 3 . In Section 4 , we use this construction to give the first example of a computable field possessing an infinite, intrinsically computable transcendence basis.

We wish to make note here of unpublished work by Kudinov and Lvov. Working jointly, they addressed the same question, about computable categoricity for fields of infinite transcendence degree, and made significant progress on it. Like us, they combined techniques from algebraic geometry and computability theory, but their investigations were unfortunately cut short when Lvov passed away, and it has not been possible to reconstruct their work. As we understand it, their constructions did not make use of the Fermat polynomials - which suggests that there are alternative ways to approach this problem, awaiting discovery (or re-discovery) by an enterprising researcher. We salute the efforts of Kudinov and Lvov, and regret the demise of the latter.

We describe our principal conventions for this paper. A computable field is a structure in the signature with addition and multiplication, whose domain is an initial segment of $\omega$, and for which those two operations are computable and define a field. This conforms to the usual computable-model-theoretic definition of a computable structure. An introduction to such fields for non-logicians is given in [25] and continued in [26]. Classic references on computable fields include [5], [6], [7], [8], [20], [23], [30], and [34]. Our computability-theoretic notation is standard and can be found in [33]. 
The field $\mathbb{Q}$ is known to be computably categorical, and so we will often just write $\mathbb{Q}$ to denote a computable presentation of that field, without concern for the specifics of the presentation. Given a computable field $F$, its polynomial ring $F[X]$ may be viewed just as the set $F^{*}$ of finite tuples of elements of $F$, with $\left\langle a_{0}, \ldots, a_{d}\right\rangle$ identified with $\sum a_{i} X^{i}$. (For a perfect identification, ensure that if $a_{d}=0$, then $d=0$.) Iterating this process yields a computable presentation of the ring $F\left[X_{1}, \ldots, X_{n}\right]$, uniformly in $n$.

Given a computable field $F$, we will treat any singly-generated field extension $F(x)$ as a computable field as well. To compute it, we will need to know whether $x$ is algebraic over $F$ or not, and if it is, we will need its minimal polynomial $p(X)$ over $F$. In the algebraic case, one views elements of $F(x)$ as $F$-linear combinations over the set $\left\{1, x, x^{2}, \ldots, x^{d-1}\right\}$, where $d=\operatorname{deg}(p)$, with the obvious addition and multiplication (which requires knowledge of $p(X)$, of course). In the transcendental case, $F(x)$ is just the quotient field of the domain $F[X]$ given above, and this quotient field is computably presentable as the Cartesian product $F[x] \times(F[x]-\{0\})$ modulo a computable equivalence relation. We can iterate these extensions, even over infinitely many generators, as long as the minimal polynomial (or lack thereof) for each generator over the preceding ones is given effectively. Notice that the base field $F$ is a computable subfield of each extension built this way.

\section{Results from algebraic geometry}

We now introduce some notation, review some algebraic geometry, and prove the results from this topic which will be needed in the subsequent sections. Since these results are nontrivial, we will assume a significant algebraic background; full explanation would require much more space. Hartshorne's book [16] provides a comprehensive description of the subject.

Let $k$ be a field of characteristic zero and fix an algebraic closure $\bar{k}$ of $k$. A variety $V$ over $k$ is by definition an absolutely irreducible, separated, reduced scheme of finite type over $k$. Recall that $V$ is called absolutely irreducible if the base change $V \times{ }_{k} \bar{k}$, (or more correctly, the fiber product $V \times \operatorname{Speck}$ Spec $\bar{k}$ ) is irreducible. In fact, $V \times{ }_{k} K$ is then irreducible for any extension $K$ of $k$.

Suppose first that $V$ is affine, that is to say, a closed subscheme of affine space $\mathbb{A}_{k}^{n}$ for some $n$, or equivalently, a scheme of the form $V=\operatorname{Spec} A$ with $A$ an absolutely irreducible (meaning that $A \otimes_{k} \bar{k}$ is a domain), finitely generated $k$-algebra. For any extension field $K$ of $k$, we define the $K$-rational points of $V$, denoted $V(K)$, as the collection of all $P \in \mathbb{A}_{K}^{n}(K)=K^{n}$ such that $P$ lies on $V$ (more precisely, in scheme-theoretic terms, this means that the morphism Spec $K \rightarrow \mathbb{A}_{K}^{n}$ induced by $P$ factors through $V$, yielding a morphism $\operatorname{Spec} K \rightarrow V$; or equivalently, that $P$ is given by a maximal ideal $\mathfrak{m}_{P}$ in $A$ such that $A / \mathfrak{m}_{P} \cong K$ ). We define the coordinate ring $k[V]$ of $V$ over $k$ as the residue ring $k\left[x_{1}, \ldots, x_{n}\right] / I_{V}$, where $x_{i}$ are variables and $I_{V}$ is the ideal of all polynomials vanishing on $V$ (that is to say, the collection of all $f \in k\left[x_{1}, \ldots, x_{n}\right]$ such that $f(P)=0$ for all $P \in V(\bar{k})$ ). Hence, in the above notation, $A \cong k[V]$. Since we assumed $V$ to be irreducible, $I_{V}$ is a prime ideal and remains so when extended to $\bar{k}\left[x_{1}, \ldots, x_{n}\right]$; we call such an ideal absolutely prime. Conversely, if $I$ is an absolutely prime ideal in $k\left[x_{1}, \ldots, x_{n}\right]$, the variety defined by $I$ is the scheme $V:=\operatorname{Spec}\left(k\left[x_{1}, \ldots, x_{n}\right] / I\right)$; for any extension $k \subseteq K$, its $K$-rational points are precisely those $P \in K^{n}$ such that $f(P)=0$ for all $f \in I$. This establishes a one-one correspondence between (affine) subvarieties of $\mathbb{A}_{k}^{n}$ and absolutely prime ideals in $k\left[x_{1}, \ldots, x_{n}\right]$ (which in turn are in one-one correspondence with the absolutely irreducible, finitely generated $k$-algebras).

We define the function field $k(V)$ of $V$ over $k$ as the field of fractions of $k[V]$. The function field of a variety is a so-called birational invariant, meaning that it only depends on the birational class of $V$. In particular, by resolution of singularities in characteristic zero, we may therefore assume, when studying the function field, that the variety has no singularities. The following quantities are all equal and are called the dimension of $V$ :

- the transcendence degree of $k(V)$ over $k$;

- the least number of hypersurfaces (=variety defined by a single, absolutely irreducible equation) $H_{1}, \ldots, H_{d}$ such that $V(\bar{k}) \cap H_{1}(\bar{k}) \cap \cdots \cap H_{d}(\bar{k})$ is non-empty and finite;

- the combinatorial dimension of $V(\bar{k})$ viewed as a topological space via its Zariski topology;

- the Krull dimension of $k[V]$. 
A point $P$ on $V$ is called generic over $k$ if the field $k(P)$ generated by its coordinates is isomorphic to the function field of $V$, or equivalently, if the transcendence degree of $k(P)$ over $k$ is equal to the dimension of $V$. Conversely, any point $P=\left(p_{1}, \ldots, p_{n}\right) \in K^{n}$ in some extension field $K \supseteq k$ can be viewed as the generic point of an affine variety $V$ over $k$ : let $\mathfrak{m}_{P}$ be the maximal ideal in $K\left[x_{1}, \ldots, x_{n}\right]$ generated by the linear polynomials $X_{i}-p_{i}$, let $I_{V}$ be the contraction of this ideal to $k\left[x_{1}, \ldots, x_{n}\right]$, and let $V$ be the affine variety with coordinate ring $k[V]:=k\left[x_{1}, \ldots, x_{n}\right] / I_{V}$. One verifies that $k(V)$ is isomorphic with the field $k(P)$, that is to say, $P$ is a generic point of $V$. If $V$ is not affine, then its function field can still be defined as the function field $k\left(V_{0}\right)$ of any non-empty affine open subset $V_{0}$ of $V$. In particular, when studying the function field, we may take the variety to be projective as well. On occasion, we will use the following simple observation:

Lemma 2.1. If $V$ is a variety over $k$, then $k$ is algebraically closed inside $k(V)$.

Proof. By assumption $V \times_{k} \bar{k}$ is irreducible, showing that $k(V) \otimes_{k} \bar{k}$ is equal to the function field $\bar{k}(V)$ of $V$ over $\bar{k}$. Towards a contradiction, assume $k \subsetneq l \subseteq k(V)$ is a finite extension of degree $d>1$. Hence $l \otimes_{k} \bar{k} \cong \bar{k}^{d}$ is not a field, which contradicts that $l \otimes_{k} \bar{k} \subseteq k(V) \otimes_{k} \bar{k}$.

\subsection{Curves}

By a curve over $k$ we will mean in this article a non-singular (also called smooth), projective one-dimensional variety $C$ over $k$. Recall that for any one-dimensional variety $X$ defined over $k$, there exists a unique curve $C$ to which $X$ is birationally equivalent, and moreover, if $X$ has no singularities, then it is isomorphic to an open subset of $C$. For instance, one can define a scheme structure on the set $C_{X}$ of discrete valuations of $k(X)$ which are trivial on $k$, and then show that $C_{X}$ is a curve birational to $X$ (see for instance [18, Theorem 7.3.1] or [16, I. Corollary 6.11]); alternatively, we can take a completion $\bar{X}$ of $X$ (e.g., the Zariski closure of $X$ viewed as a subvariety of some projective space over $k$ ), and then take its normalization. A one-dimensional variety over $k$ which is birational to a curve $C$ over $k$ is sometimes called a model of $C$. Recall (see for instance [16, IV. Corollary 3.11]) that any curve admits an affine plane model (with at most nodes as singularities). By Lemma 2.1, a point $P$ on $C$ is generic (over $k)$ if and only if $P \notin C(\bar{k})$.

One can associate to any curve $C$ a natural number $g(C)$, called its genus, which is a birational invariant and does not change when extending $k$ to a larger base field. In case $C$ is a plane curve (that is, a curve in $\mathbb{P}_{k}^{2}$ ), then its genus is calculated as $g(C):=(d-1)(d-2) / 2$, where $d$ is the degree of the defining equation of $C$ (see for instance [16, I. Exercise 7.2]).

Any morphism between curves $C \rightarrow D$ defined over $k$ is either constant (that is to say, its image is a single point, necessarily $k$-rational), or otherwise surjective. In the latter case, we have an inclusion of function fields $k(D) \subseteq k(C)$, which is necessarily a finite extension, since both $k(C)$ and $k(D)$ have transcendence degree one over $k$. It follows that $C \rightarrow D$ is a finite morphism of degree $d:=((k(C): k(D))$; in particular, all the fibers of $C \rightarrow D$ are finite, of cardinality at most $d$.

The following fact will be quite useful.

Proposition 2.2. Let $C$ be a curve and $V$ any variety, both defined over a field $k$. We have a functorial bijection of sets

$$
\operatorname{Mor}_{k}(C, V) \cong V(k(C)),
$$

and under this bijection, the morphism $C \rightarrow V$ is constant if and only if the associated $k(C)$-rational point of $V$ is k-rational.

Proof. Let $P$ be a $k(C)$-rational point on $V$, which therefore corresponds to a morphism $\operatorname{Spec} k(C) \rightarrow V$. By the valuative criterion for properness (see for instance [16, I. Proposition 6.8 or II. Theorem 4.7] or [18, Proposition 7.2.3]), this extends to a morphism $C \rightarrow V$. Conversely given a morphism $v: C \rightarrow V$, choose affine open sets $C_{0} \subseteq C$ and $V_{0} \subseteq V$ such that $v\left(C_{0}\right) \subseteq V_{0}$. Hence we have an induced $k$-algebra homomorphism $k\left[V_{0}\right] \rightarrow k\left[C_{0}\right]$. Composing this with the inclusion $k\left[C_{0}\right] \subseteq k\left(C_{0}\right)=k(C)$ then yields a $k(C)$-rational point $\operatorname{Spec} k(C) \rightarrow V$. 
Functoriality here means that we can view $\operatorname{Mor}_{k}(\cdot, V)$ and $V(\cdot)$ as contravariant functors, and the above bijection is compatible with these functors in the following sense. Given a non-constant morphism $C \rightarrow D$, composition yields a map $\operatorname{Mor}_{k}(D, V) \rightarrow \operatorname{Mor}_{k}(C, V)$. Moreover, we have an extension $k(D) \subset k(C)$ of function fields, giving rise to an inclusion $V(k(D)) \subset V(k(C)$. One now easily checks that we have a commutative diagram

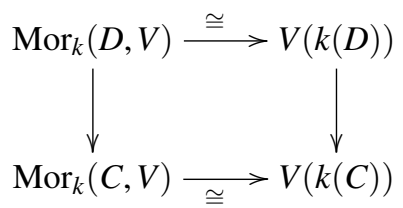

Moreover, these bijections are also compatible when viewed as (covariant) functors in their second component, that is to say, given a non-constant morphism $V \rightarrow W$, composition yields $\operatorname{Mor}_{k}(C, V) \rightarrow \operatorname{Mor}_{k}(C, W)$, and we get a natural map $V(k(C)) \rightarrow W(k(C))$, making the diagram

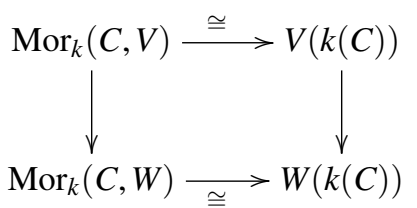

commute as well.

Moreover, if $V$ is actually a curve, then by our above discussion, under this isomorphism, the morphism $C \rightarrow V$ is constant if and only if the associated $k(C)$-rational point of $V$ is in fact $k$-rational.

Given two curves $C$ and $D$ over $k$, we say that $C$ covers (or dominates) $D$ if there exists a non-constant morphism $C \rightarrow D$ defined over some field extension $K \supseteq k$. Note that such a $C \rightarrow D$ is then automatically finite and surjective. By Proposition 2.2, therefore, $C$ does not cover $D$ if and only if $\operatorname{Mor}_{K}(C, D) \cong D(K)$ for every $K \supseteq k$.

Lemma 2.3. Let $C$ and $D_{i}$ be curves over $k$ such that no $D_{i}$ covers $C$. If $F$ is the field generated by all the function fields $k\left(D_{i}\right)$, then $C(F)=C(k)$.

Proof. The essence of the proof is to build $F$ not all at once from the fields $k\left(D_{i}\right)$, but rather by induction. Let $F_{i}$ be the subfield of $F$ generated by all $k\left(D_{j}\right)$ with $j \leq i$, that is to say, $F_{i}$ is defined inductively as the function field $F_{i-1}\left(D_{i}\right)$ of the curve $D_{i}$ viewed as a curve over $F_{i-1}$ (with $F_{0}:=k$ ). It suffices to show by induction on $i$ that $C\left(F_{i}\right)=C(k)$. Suppose $P$ is an $F_{i}$-rational point on $C$, and we want to show that $P$ is $k$-rational. Since $P \in C\left(F_{i-1}\left(D_{i}\right)\right)$, we get from Proposition 2.2 a morphism $D_{i} \rightarrow C$ defined over $F_{i-1}$. Since $D_{i}$ does not cover $C$, this morphism must be constant, showing that $P$ belongs to $C\left(F_{i-1}\right)$ and by induction, the latter is just $C(k)$.

We will also use the following well-known inequality (see for instance [16, Corollary 2.4]), which leads to a quick proof of Proposition 2.5 under the assumption that the genus is at least 2. (Our construction in Section 3 will require a genus $\geq 2$ in any case.)

Theorem 2.4. Let $k$ be an algebraically closed field of characteristic zero and let $C$ and $D$ be curves of genus $g(C)$ and $g(D)$ respectively. If $C \rightarrow D$ is a finite morphism of degree $d$, then $g(C)-1 \geq d \cdot(g(D)-1)$.

Proof. Since the characteristic is zero, the morphism is separable, so that Hurwitz's Formula yields

$$
2(g(C)-1)=2 d(g(D)-1)+\operatorname{deg}(R),
$$

where $R$ is the (effective) ramification divisor of $C \rightarrow D$; see for instance [16, IV. Corollary 2.4]. 
Proposition 2.5. Let $k$ be a field of characteristic zero and let $C$ be a curve over $k$ of genus $g \geq 2$. Then the function field $K:=k(C)$ of $C$ is generated by the coordinates of any $K$-rational point $P$ of $C$ which is not $k$-rational, that is to say, for any $P \in C(K) \backslash C(k)$, the natural inclusion $k(P) \subseteq K$ is an equality.

Proof. Let $C_{0}$ be a (non-empty) affine open subset of $C$ and let $A:=k\left[C_{0}\right]$ be its coordinate ring. For instance, if $C_{0}$ is a plane curve with affine equation $f=0$, then $A=k[x, y] / f k[x, y]$ and $K$ is the field of fractions of $A$. We need to show that if $P:=(a, b) \in K^{2} \backslash k^{2}$ satisfies $f(a, b)=0$, then $k(P):=k(a, b)$ is equal to $K$. In any case, since $P$ is not defined over $k$, it is a generic point of $C$ by Lemma 2.1. In particular, $k(P)$ and $K$ are isomorphic over $k$ (for instance, in the planar example, the $k$-algebra $k[a, b]$ is easily seen to be $k$-isomorphic to $A)$. In particular, by our discussion of Proposition 2.2, the embedding $k(P) \subseteq K$ is finite of degree $d:=(K: k(P))$ inducing a finite morphism $C \rightarrow C$. We need to show that $d=1$.

Assume first that $k$ is algebraically closed, so that we can apply Theorem 2.4 to this morphism, giving $g-$ $1 \geq d(g-1)$. Since $g \geq 2$, we must have $d=1$, as we wanted to show. For the general case, it follows from the algebraically closed case that there exists a finite extension $l$ of $k$ such that $l(P)=l(C)$. Let $\pi$ be such that $l=k(\pi)$, so that in particular $l(P)=k(P, \pi)$ and $l(C)=K(\pi)$. However, since $\pi$ is algebraic over $k$ whence over the $k$-isomorphic fields $k(P)$ and $K$, the degrees $(l(P): k(P))$ and $(l(C): K)$ are the same. By transitivity, it follows that $(K: k(P))=1$, as we wanted to show.

Remark 2.1. The proposition is false in positive characteristic $p$, precisely because of the purely inseparable extenstion given by the Frobenius Frob F $_{p}$ if $P$ is a $K$-rational point of $C$, then so is its Frobenius transform $\operatorname{Frob}_{p}(P)$, which clearly generates a proper subfield.

Remark 2.2. By the argument in the proof, $g(C) \geq g(D)$ for any cover $C \rightarrow D$ over $k$ (not necessarily algebraically closed). Moreover, if these genera are equal and $\geq 2$, then $C \rightarrow D$ must have degree one, hence is an isomorphism. In summary, given a cover $C \rightarrow D$ of curves of genus at least two, either $C \cong D$ (and the cover itself is an isomorphism) or $g(C)>g(D)$.

Remark 2.3. Let $C$ be a curve of genus $g$ at least two and let $K:=k(C)$ be its function field. Any non-constant morphism $C \rightarrow C$ is necessarily an automorphism by our previous remark. Hence under Proposition 2.2, we have a one-one correspondence between the $K$-rational generic points of $C$ and the automorphisms of $C$. In particular, $C(K) \backslash C(k)$ has cardinality at most $84(g-1)$, as this is the maximum number of automorphisms of $C$ (see for instance [16, IV Exercise 2.5]). In particular, if $C$ has no non-trivial automorphism (which is the 'generic' case for $g \geq 3$ ), then $C$ has a unique generic point (in any fixed function field).

\subsection{General collections of curves}

By a general collection of curves over $k$, we mean a countable set $\mathscr{C}$ of ( $k$-isomorphism classes of ) curves over $k$ of genus at least two, such that no two distinct curves in $\mathscr{C}$ are isomorphic over $\bar{k}$. The function field $k(\mathscr{C})$ of $\mathscr{C}$ is by definition the field generated by all the function fields of curves in $\mathscr{C}$. More precisely, take a universal field $\Omega$ (that is, an algebraically closed field containing $k$ and of cardinality larger than any of the fields we use otherwise), and for each $C \in \mathscr{C}$, fix a subfield $k_{C} \subseteq \Omega$ isomorphic to its function field $k(C)$. Then $k(\mathscr{C})$ is the subfield of $\Omega$ generated by all the $k_{C}$ with $C \in \mathscr{C}$. Note that the field generated by the subfields $k_{C_{1}}, \ldots, k_{C_{i}}$ is isomorphic to the function field of the product variety $C_{1} \times \cdots \times C_{i}$, for $C_{1}, \ldots, C_{i}$ distinct curves in $\mathscr{C}$. Moreover, $k(\mathscr{C})$ is the union of the function fields of all such products. In particular, $k(\mathscr{C})$ is well-defined up to isomorphism.

Proposition 2.6. Let $\mathscr{C}$ be a general collection of curves over $k$ and let $k(\mathscr{C})$ be its function field. Suppose all curves in $\mathscr{C}$ have genus at most $g$ and let $D$ be an arbitrary curve of genus at least $g$. Then the function field $k(D)$ embeds in $k(\mathscr{C})$ if and only if $D$ is isomorphic to some curve in $\mathscr{C}$.

Proof. Let us write $k_{D}$ for the image of $k(D)$ in $k(\mathscr{C})$. Let $C_{1}, C_{2}, \ldots$ be an enumeration of $\mathscr{C}$, and let $k_{i}:=k\left(C_{1} \times\right.$ $\left.\cdots \times C_{i}\right) \subseteq k(\mathscr{C})$ be as above, so that in particular $k(\mathscr{C})$ is the union of all $k_{i}$. Since $k_{D}$ is finitely generated, it lies in some $k_{i}$. Let $i$ be minimal such. By Proposition 2.5 and minimality of $i$, the function field $k_{i-1}(D)$ of $D$ over $k_{i-1}$ is 
isomorphic to the field generated by $k_{i-1}$ and $k_{D}$, and hence has transcendence degree one over $k_{i-1}$ by Lemma 2.1 . The extension $k_{i-1}(D) \rightarrow k_{i}=k_{i-1}\left(C_{i}\right)$ is finite, since both fields have transcendence degree one over $k_{i-1}$, and hence by the discussion of Proposition 2.2, determines a cover $C_{i} \rightarrow D$ over $k_{i-1}$. If $C_{i} \not D D$, then $g\left(C_{i}\right)>g(D) \geq g$ by Remark 2.2, contradicting the assumption that $g\left(C_{i}\right) \leq g$.

We say that a general collection of curves $\mathscr{C}$ is non-covering, if there is no cover relation between any two distinct curves in $\mathscr{C}$. Immediately from Remark 2.2 we get:

Lemma 2.7. The collection of all isomorphism classes of curves over $k$ of a fixed genus $g \geq 2$ is non-covering.

Note that the collection in Lemma 2.7 is in one-one correspondence with the set of $k$-rational points of the moduli space $\mathscr{M}_{g}$ of all curves of genus $g$. Recall that the moduli space has dimension $3(g-1)$, for $g \geq 2$.

Theorem 2.8. Let $\mathscr{C}$ be a non-covering collection of curves over $k$, and let $k(\mathscr{C})$ be its function field, as in Proposition 2.6. For any curve $C$ in $\mathscr{C}$, any embedding of its function field into $k(\mathscr{C})$ has image equal to $k_{C}$. In particular, if $P$ is a $k(\mathscr{C})$-rational point on $C$ which is not $k$-rational, then $P$ is $k_{C}$-rational and the natural inclusion $k(P) \subseteq k_{C}$ is an equality.

Proof. Let $K$ be the subfield of $k(\mathscr{C})$ generated by all the function fields $k_{D}$ with $D \in \mathscr{C}$ different from $C$. Since $\mathscr{C}$ is non-covering, we can apply Lemma 2.3 over the subfield $k_{C} \subseteq k(\mathscr{C})$. Since $k(\mathscr{C})$ is generated by $K$ and $k_{C}$, Lemma 2.3 shows that $P$ is $k_{C}$-rational. As $P$ is not $k$-rational, Proposition 2.5 then shows that $k(P)=k_{C}$, as we wanted to show. To prove the first assertion, let $k(C) \rightarrow k(\mathscr{C})$ be an embedding, and let $P$ be the corresponding generic point of $C$ defined over $k(\mathscr{C})$. In particular, $k(P)$ is the image of the above embedding, and we just argued that $k(P)=k_{C}$.

Corollary 2.9. Let $\mathscr{C}$ be a non-covering collection of curves over $k$, and let $k(\mathscr{C})$ be its function field. Then the Galois group $G$ of $k(\mathscr{C})$ over $k$ is equal to the direct product of the automorphism groups of each curve in $\mathscr{C}$. In particular, if all curves in $\mathscr{C}$ have genus at most $g$, then $G$ has exponent at most $84(g-1)$.

Proof. Let $C$ be a curve in $\mathscr{C}$ with function field $k_{C} \subseteq k(\mathscr{C})$, and let $g \in G$. Since $g\left(k_{C}\right) \cong k_{C}$, it must be equal to it by Theorem 2.8. In other words, the restriction of $g$ to $k_{C}$ belongs to the Galois group $H_{C}$ of $k_{C}$ over $k$. In particular, the restriction map induces a split group homomorphism $G \rightarrow H_{C}$. Since $k(\mathscr{C})$ is generated by all the $k_{C}$ with $C \in \mathscr{C}$, it follows that $G$ is isomorphic to the direct product of all $H_{C}$. Since $H_{C} \cong \operatorname{Aut}_{k}(C)$ has order at most $84(g(C)-1)$, the result follows (see Remark 2.3).

\section{Construction of a computably categorical field of infinite transcendence degree}

In this section, $Q$ is a (countable) computable field of characteristic zero. Given a collection $\mathscr{C}=\left\{C_{0}, C_{1}, \ldots\right\}$ of curves $C_{i}$ over $Q$, we say that $\mathscr{C}$ has the effective Faltings property (or is effectively Faltings) if the values $\left|C_{i}(Q)\right|<\infty$ are computable as a function of $i$. Of course, this depends not only on $\mathscr{C}$, but also on the ordering of the curves in $\mathscr{C}$. Normally we assume a fixed computable listing of these curves: some computable function $g(i)$ gives the defining equation of each $C_{i}$ over $Q$. One might hope to compute each $\left|C_{i}(Q)\right|$ effectively while still allowing $\left|C_{i}(Q)\right|=\aleph_{0}$, but in practice we will deal with collections $\mathscr{C}$ with the basic Faltings property, i.e., for which all $\left|C_{i}(Q)\right|$ are finite. In this case, with a computable ground field $Q$ and a computable listing of the curves in $\mathscr{C}$, the effective Faltings property for $\mathscr{C}$ means that we can computably determine the entire set $C_{i}(Q)$ of solutions for each $i$, since to do so we only need to be able to compute their number, using the effectiveness of the Faltings property, and then search for them all. Note that by Faltings's positive solution of the Mordell Conjecture, each curve of genus two has only finitely many solutions over a number field. (The term "Mordell Conjecture" is still widely used, but in addition to being an inapt name for a proven theorem, this nomenclature seems to slight Faltings, who finally proved it. We chose the name "Faltings property" in part to try to allot more credit to Faltings.)

For our construction, we need a non-covering collection of curves over the ground field $Q=\mathbb{Q}$ with the effective Faltings property. For instance, we might in light of Lemma 2.7 ask whether there are infinitely many curves of a 
fixed genus for which we can effectively determine their $\mathbb{Q}$-rational points. After all, a "generic" choice will produce a curve without any $\mathbb{Q}$-rational points, a trivial instance of effective Faltings. Although we do not know the answer to this, we can prove:

Theorem 3.1. There exists a non-covering collection $\mathscr{C}$ of curves over $\mathbb{Q}$ with the effective Faltings property.

Proof. Our collection will consist of Fermat curves, which is to say, plane curves $D_{N}$ with affine equation $d_{N}(X, Y)=X^{N}+Y^{N}-1$. By Wiles's positive solution of Fermat's Theorem, the set of Fermat curves $D_{N}$ with $N \geq 3$ is effectively Faltings, since the only $\mathbb{Q}$-rational points of such a curve are $(1,0)$ and $(0,1)$ (and the point at infinity $(1:-1: 0)$ ). Moreover, the genus of $D_{N}$ is equal to $(N-1)(N-2) / 2$. So it remains to find a non-covering subset. To this end, we will choose inductively a set of prime exponents $p_{0}, p_{1}, \ldots$ as follows. Let $p_{0}=5$, and choose $p_{i+1}$ to be the least prime bigger than $\left(4\left(p_{i}-1\right)\left(p_{i}-2\right)\right)^{2}$. Now $D_{p_{i}}$ does not cover $D_{p_{j}}$ for $i>j$ by Lemma 3.3 below, and for $i<j$ by Remark 2.2.

To prove Lemma 3.3, we need the following result from [1, Lemma 9.3], using the explicit value $\operatorname{gcd}(m !, N)$ for $N^{\prime}$ which they give there.

Lemma 3.2 (Baker, González-Jiménez, González \& Poonen). Let $C$ be a curve of genus $g \geq 2$ and suppose $m>180$ has the property that $\varphi(n)>8 g$ for all $n>m$, where $\varphi$ is Euler's totient function. If $C$ is dominated by some Fermat curve $D_{N}$, then it is already dominated by the Fermat curve $D_{N^{\prime}}$ where $N^{\prime}=\operatorname{gcd}(m !, N)$.

Lemma 3.3. Let $C$ be a curve of genus $g \geq 2$ and let $D_{p}$ be the Fermat curve of prime exponent $p$. If $p>64 g^{2}$, then there is no cover relation between $C$ and $D_{p}$.

Proof. Since $D_{p}$ has genus $(p-1)(p-2) / 2>g$, it cannot be covered by $C$ by Remark 2.2 . We verify that $m=64 g^{2}$ satisfies the hypotheses of Lemma 3.2. Let $n>64 g^{2}$. By the well-known estimate for the Euler's totient function $\varphi(n) \geq \sqrt{n}$, we get $\varphi(n)>8 g$. Since $p$ does not divide $\left(64 g^{2}\right)$ !, there can be no covering $D_{p} \rightarrow C$.

Fixing the sequence of primes $\left\langle p_{s}\right\rangle_{s \geq 0}$ defined in Theorem 3.1, we begin with the purely transcendental extension $\mathbb{Q}\left(x_{0}, x_{1}, \ldots\right)$, and for each $s$, adjoin an element $y_{s}$ satisfying the $p_{s}$-th Fermat curve $d_{p_{s}}\left(x_{s}, y_{s}\right)=x_{s}^{p_{s}}+y_{s}^{p_{s}}-1=0$. We write $F_{0}=\mathbb{Q}$ and $F_{s+1}=F_{s}\left(x_{s}\right)\left[y_{s}\right] /\left(d_{p_{s}}\left(x_{s}, y_{s}\right)\right)$, so $F_{s+1}$ has transcendence degree 1 over $F_{s}$, and we may take each $F_{s}$ to be computable within $F$, uniformly in $s$. Clearly $F$ has infinite transcendence degree over $\mathbb{Q}$.

Theorem 3.4. The computable field $F$ built above is computably categorical and has infinite transcendence degree.

Proof. Let $\tilde{F}$ be any computable field isomorphic to $F$, say via a noncomputable isomorphism $\psi: F \rightarrow \tilde{F}$. We define our computable isomorphism $f$ on each $F_{s}$ in turn, starting with the unique (and computable) embedding of the rationals $F_{0}$ onto the prime subfield $\tilde{F}_{0}$ of $\tilde{F}$. Given $f\left\lceil F_{s-1}\right.$, search for any nonzero elements $\tilde{x}$ and $\tilde{y}$ in $\tilde{F}$ such that $d_{p_{s}}(\tilde{x}, \tilde{y})=0$ there. We must find them, since $F \cong \tilde{F}$, and $(\tilde{x}, \tilde{y})$ is then a generic point of $D_{p}$ over $\mathbb{Q}$. So we define $f\left(x_{s}\right)=\tilde{x}$ and $f\left(y_{s}\right)=\tilde{y}$, then extend this $f$ to the rest of $F_{s}$, which is generated over $F_{s-1}$ by these elements. This gives a field embedding of $F_{s}$ into $\tilde{F}$, whose image is shown by Theorem 2.8 to be uniquely determined as the subfield $\psi\left(F_{S}\right)$ (although the embedding itself may not equal $\psi$ on $F_{s}$ ). So the union of these embeddings (for all $s$ ) must map $F$ onto $\tilde{F}$.

One could begin with any prime $>3$ as $p_{0}$, thereby building countably many non-isomorphic computable, computably categorical fields of infinite transcendence degree. (Our use of Proposition 2.5 requires that $p_{0} \neq 3$, since the curves must all have genus $\geq 2$.) And by varying the choice of the subsequent primes $p_{1}, p_{2}, \ldots$, one could get uncountably many relatively computably categorical fields of infinite transcendence degree, although of course only countably many of them would be computably presentable.

Next we adapt Theorem 3.4 to a more general setting.

Theorem 3.5. Let $k$ be any finitely generated field of characteristic 0 , and $\mathscr{C}$ any computably enumerable collection of curves over $k$, all of genus $\geq 2$, such that $\mathscr{C}$ is non-covering and effectively Faltings over $k$. (In particular, $C(k)$ is finite for all $C \in \mathscr{C}$.) Then the function field $k(\mathscr{C})$ is computably categorical. 
Saying that $\mathscr{C}$ is computably enumerable (or c.e.) means that there is a computable function $g$ such that for every $s, g(s) \in k[X, Y]$ is a polynomial defining a curve $C_{s}$, and $C_{0}, C_{1}, \ldots$ is a list of all curves in $\mathscr{C}$ without repetitions (even up to isomorphism). Below we write $g_{s}$ for $g(s)$.

Proof. First, since $k$ is finitely generated, it has a computable presentation. Then, by enumerating the curves in $\mathscr{C}$ and adjoining to $k$ a generic solution for each, we may build a computable field which, by Theorem 2.8 , is isomorphic to $k(\mathscr{C})$. Thus $k(\mathscr{C})$ is computably presentable.

Now let $F$ and $\tilde{F}$ be computable fields isomorphic to $k(\mathscr{C})$. The finitely generated field $k$ is computably enumerable within $F$, and for a fixed (not necessarily computable) isomorphism $\alpha: F \rightarrow \tilde{F}$, the image $\tilde{k}=\alpha(k)$ will likewise be c.e. within $\tilde{F}$. Moreover, the isomorphism $f_{0}=\alpha \nmid k$ from the subfield $F_{0}=k$ onto $\tilde{F}_{0}=\tilde{k}$ is computable: we need only know the images under $\alpha$ of the finitely many generators of $k$.

As in Theorem 3.4, the construction is straightforward. For each $s$, using the effective Faltings property, we can compute the (finite) number $j$ of distinct solutions of $g_{s}$ in $k$. Search until we have found all $j$ solutions of $g_{s}$ in $k$ and one additional solution $\left(x_{s}, y_{s}\right)$ of $g_{s}$ in $F$. Then we search in $\tilde{k}$ for $j$ distinct solutions to the polynomial $\tilde{g}_{s}(X, Y) \in \tilde{k}[X, Y]$ whose coefficients are the images of those of $g_{s}$ under $f_{0}$, and in $\tilde{F}$ for one additional solution $\left(\tilde{x}_{s}, \tilde{y}_{s}\right)$ to $\tilde{g}_{s}$. We must find such a pair $\left(\tilde{x}_{s}, \tilde{y}_{s}\right)$, and we set $f\left(x_{s}\right)=\tilde{x}_{s}$ and $f\left(y_{s}\right)=\tilde{y}_{s}$. It is then easy to compute $f$ on all of $F$, since Theorem 2.8 shows $F$ to be generated by $\left\{x_{s}, y_{s}: s \in \omega\right\}$. For any $z \in F$, we compute $f(z)$ just by searching for an $n$ and an $h \in k\left(X_{1}, \ldots, X_{n}, Y_{1}, \ldots, Y_{n}\right)$ with $h\left(x_{1}, \ldots, x_{n}, y_{1}, \ldots, y_{n}\right)=z$, since then $f(z)=h\left(\tilde{x}_{1}, \ldots, \tilde{x}_{n}, \tilde{y}_{1}, \ldots, \tilde{y}_{n}\right)$.

To see that this $f$ really is an isomorphism, we appeal to the following lemma. In Theorem 3.4 , the corresponding fact is immediate (and appears explicitly in the discussion of the proof of Proposition 4.5 below). Now in this more general situation, we make sure that it is safe to choose an arbitrary solution $\left(\tilde{x}_{s}, \tilde{y}_{s}\right)$ of $\tilde{g}_{s}$ in $(\tilde{F}-\tilde{k})$.

Lemma 3.6. For every $s$ and any pairs $(x, y),\left(x^{\prime}, y^{\prime}\right) \in\left(F_{s+1}-F_{s}\right)^{2}$ with $g_{s}(x, y)=g_{s}\left(x^{\prime}, y^{\prime}\right)=0$, every automorphism $\psi$ of $F_{s}$ which fixes $k$ pointwise extends to a unique automorphism of $F_{s+1}$ which maps $x$ to $x^{\prime}$ and $y$ to $y^{\prime}$.

Proof. By Lemma 2.1, $F_{s}$ is algebraically closed within $F_{s}\left(C_{s}\right)=F_{s+1}$, so each of $x$ and $x^{\prime}$ must be transcendental over $F_{s}$. Therefore $\psi$ extends to a partial automorphism $\psi^{\prime}$ of $F_{s+1}$ by mapping $x$ to $x^{\prime}$. Next, by absolute irreducibility, $g_{s}(x, Y)$ is irreducible in $F_{s}(x)[Y]$, and likewise for $x^{\prime}$, so

$$
F_{s}(x, y) \cong F_{s}(x)[Y] /\left(g_{s}(x, Y)\right) \cong F_{s}\left(x^{\prime}\right)[Y] /\left(g_{s}\left(x^{\prime}, Y\right)\right) \cong F_{s}\left(x^{\prime}, y^{\prime}\right)
$$

with the middle isomorphism being induced by $\psi^{\prime}$ on the quotients of the polynomial rings. (It is important here that $\psi^{\prime}$ fixes the coefficients of $g_{s}(X, Y)$.) But by Theorem 2.8, $x$ and $y$ together generate $F_{s+1}$ over $F_{s}$, as do $x^{\prime}$ and $y^{\prime}$, which makes it clear both that we have an automorphism of $F_{s+1}$, and that it is unique.

Our claim that the $f=\cup_{s} f_{s}$ constructed above is an isomorphism will follow from $\left(f^{-1} \circ \alpha\right)$ being an automorphism of $F$, where $\alpha$ was the given isomorphism from $F$ to $\tilde{F}$. Since $f_{0}=\alpha\left\lceil k\right.$, we know that $\left(f_{0}^{-1} \circ \alpha\right)$ is the identity on $k=F_{0}$. For each $s,\left(f^{-1} \circ \alpha\right)\left\lceil F_{s+1}\right.$ is just the unique extension of $\psi=\left(f^{-1} \circ \alpha\right)\left\lceil F_{s}\right.$ given by the lemma, with $x=\alpha^{-1}\left(\tilde{x}_{s}\right), y=\alpha^{-1}\left(\tilde{y}_{s}\right), x^{\prime}=x_{s}$, and $y^{\prime}=y_{s}$.

We offer a criterion for computable enumerability of certain collections $\mathscr{C}$ of curves.

Theorem 3.7. Let $g \geq 2$, let $Q$ be a computable field, and let $\mathscr{C}$ be an infinite subset (indexed by natural numbers) of the $Q$-rational points of the moduli space $\mathscr{M}_{\mathrm{g}}$. Suppose for each index of a $Q$-rational point of the moduli space $\mathscr{M}_{g}$, we can effectively compute a defining set of equations over $Q$ of an affine model of the curve of genus $g$ determined by this $Q$-rational point. Then relative to a computable representation of the field extension $Q \subseteq Q(\mathscr{C})$, the subset $\mathscr{C}$ is computably enumerable inside $\mathscr{M}_{g}(Q)$.

Proof. Let $C$ be a $Q$-rational point of $\mathscr{M}_{g}$, viewed as a curve over $Q$ of genus $g$, and let $f_{1}, \ldots, f_{s} \in Q\left[x_{1}, \ldots, x_{m}\right]$ be the defining equations of an affine model of $C$ (meaning that the function field of $C$ is the field of fractions of $\left.Q\left[x_{1}, \ldots, x_{m}\right] /\left(f_{1}, \ldots, f_{s}\right) Q\left[x_{1}, \ldots, x_{m}\right]\right)$. We now search for a solution $P \in Q(\mathscr{C})^{m}$ of the system of equations $f_{1}=\cdots=f_{s}=0$ which is not defined over the computable subfield $Q$. If such a solution exists, then $C \in \mathscr{C}$ by Proposition 2.6. 
Example 3.1. For instance, if $g=2$, any curve $C$ of genus 2 has an affine model $C_{f}$ given by an equation $y^{2}=f(x)$ with $f$ a polynomial of degree 6 without double roots (so that in particular $C_{f}$ is non-singular whence isomorphic to an open subset of $C$ ). Moreover, two such models $C_{f_{1}}$ and $C_{f_{2}}$ are birational to the same curve if and only if $f_{1}$ and $f_{2}$ are equal up to a fractional linear transformation, that is to say, if and only if

$$
f_{1}(x)=(c x+d)^{6} \cdot f_{2}\left(\frac{a x+b}{c x+d}\right)
$$

for some $a, b, c, d \in Q$ with $a d-b c \neq 0$ (see for instance $[3, \S 1.1]$ ). Put differently, the subset $\mathbb{H}$ in the Hilbert scheme $\mathbb{P}_{Q}^{7}$ of degree six polynomials without a double root admits a natural action of the group of fractional linear transformations $\Gamma:=\operatorname{PGL}(2, Q)$, and the geometric quotient $\mathbb{H} / / \Gamma$ is the moduli space $\mathscr{M}_{2}$.

\section{An Intrinsically Computable Transcendence Basis}

The field $F$ built in Theorem 3.4 has uncountably many automorphisms, as one sees by defining, for any $S \subseteq \omega$, an automorphism extending the map

$$
x_{i} \mapsto\left\{\begin{array}{l}
x_{i}, \text { if } i \in S \\
y_{i}, \text { if } i \notin S
\end{array}\right.
$$

Therefore, between $F$ and any computable field $\tilde{F} \cong F$, there exist $2^{\omega}$-many isomorphisms. The point of Theorem 3.4 was that at least one must be computable, but the image of the transcendence basis $B=\left\{x_{i}: i \in \omega\right\}$ can have arbitrarily high Turing degree as well: its image under the above automorphism is Turing-equivalent to $S$. In the language of computable model theory, this says that $B$ is far from being intrinsically computable.

Definition 4.1. Let $\mathscr{M}$ be any computable structure, and $R$ an $n$-ary relation on $\mathscr{M}$ (generally not in the signature of $\mathscr{M}$ ). The degree spectrum of $R$ on $\mathscr{M}$ is the set

$$
\operatorname{DgSp}_{\mathscr{M}}(R)=\{\operatorname{deg}(f(R)): \mathscr{B} \cong \mathscr{M} \text { via } f \& \mathscr{B} \text { is a computable structure }\} .
$$

If $\operatorname{DgSp}_{\mathscr{M}}(R)=\{\mathbf{0}\}$, then $R$ is intrinsically computable.

So the degree spectrum measures the amount of complexity that can be added to (or withheld from) $R$ under isomorphisms onto other computable structures. If that amount is bounded above (or below), then we think of $R$ as being intrinsically no more complex than (or no less complex than) that bound. Degree spectra of relations have been studied widely in computable model theory; see [15] for a survey of results. With our field $F$, the degree spectrum of the basis $B$, viewed as a unary relation on $F$, contains every Turing degree, simply by the argument above using automorphisms from $F$ to itself.

In this section we show that we can go to the opposite extreme: an infinite transcendence basis for a computable field can be intrinsically computable. This would be trivial if the basis were finite, of course, but for the infinite case, we believe this is the first proof of Theorem 4.2, and more generally the first proof of Corollary 4.3 (except insofar as the corollary already follows from the proof of Theorem 3.4: every computably categorical field with an infinite computable transcendence basis instantiates the corollary).

Theorem 4.2. There exists a computable field $F$, of infinite transcendence degree over its prime field $\mathbb{Q}$, with an intrinsically computable transcendence basis.

Corollary 4.3. . There exists a computable field $F$, of infinite transcendence degree over its prime field $\mathbb{Q}$, such that every computable field isomorphic to $F$ has a computable transcendence basis.

The field $F$ in the theorem will be the computable field defined in Theorem 3.4, and the key to the proof is knowing the exact number of solutions in $F$ to each of the Fermat polynomials $d_{p_{i}}(X, Y)$ used to identify transcendence basis elements. Therefore, we need the following essential result of Tzermias and Leopoldt. 
Theorem 4.4 (Tzermias [35]; Leopoldt [22]). Over an algebraically closed field $K$ of characteristic 0 , the automorphism group of the projective curve $X^{p}+Y^{p}+Z^{p}$ is the semidirect product of the symmetric group $S_{3}$ and the group $(\mu(p))^{2}$, where $\mu(p)$ is the multiplicative group of $p$-th roots of unity in $K$.

In particular, $S_{3}$ acts by permuting the coordinates in $\mathbb{P}^{2}(K)$, and $(\alpha, \beta) \in(\mu(p))^{2}$ maps $(x: y: z)$ to $(\alpha x: \beta y: z)$. Clearly each of these operations maps solutions of $X^{p}+Y^{p}+Z^{p}=0$ to other solutions; the content of the theorem is that there are no other automorphisms.

Proposition 4.5. The function field $\mathbb{Q}\left(D_{p}\right)$ of the Fermat curve $D_{p}$ of prime exponent $p>3$ contains exactly eight solutions (given below) to its defining equation $d_{p}(X, Y)=0$.

Proof. Since $\mathbb{Q}$ contains no non-trivial $p$-th roots of unity, the automorphism group of $D_{p}$ is isomorphic to $S_{3}$, and corresponds by Remark 2.3 to the six generic points on $D_{p}$. By Wiles' positive solution to Fermat's Last Theorem, $D_{p}(\mathbb{Q})$ consists of three points, including the point at infinity $(1:-1: 0)$. Excluding the point at infinity gives a total of eight affine solutions to the equation $d_{p}=0$.

We now describe this proof more explicitly for readers with less background in algebraic geometry. The function field $\mathbb{Q}\left(D_{p}\right)$ must contain at least one nontrivial solution $(x, y)$ to $X^{p}+Y^{p}=1$. Now with $p>3, D_{p}$ has genus $\geq 2$, so if $\left(x^{\prime}, y^{\prime}\right) \in\left(\mathbb{Q}\left(D_{p}\right)\right)^{2}$ is any nontrivial solution, then $x^{\prime}$ and $y^{\prime}$ generate $\mathbb{Q}\left(D_{p}\right)$, by Proposition 2.5 (as do $x$ and $y$ also). Hence $x^{\prime}$ is transcendental over $\mathbb{Q}$, forcing $\mathbb{Q}(x) \cong \mathbb{Q}\left(x^{\prime}\right)$, which in turn yields a chain whose composition is an automorphism of $\mathbb{Q}\left(D_{p}\right)$ :

$$
\mathbb{Q}(x, y) \cong \mathbb{Q}(x)[Y] /\left(d_{p}(x, Y)\right) \cong \mathbb{Q}\left(x^{\prime}\right)[Y] /\left(d_{p}\left(x^{\prime}, Y\right)\right) \cong \mathbb{Q}\left(x^{\prime}, y^{\prime}\right) .
$$

Thus $\left(x^{\prime}: y^{\prime}:-1\right)$ gives an automorphism of the projective curve $X^{p}+Y^{p}+Z^{p}$, using Remark 2.3, and so Theorem 4.4 shows that the only projective solutions to $X^{p}+Y^{p}+Z^{p}=0$ in the function field are $(\alpha x: \beta y:-1)$ and permutations thereof, with $\alpha$ and $\beta$ ranging over the $p$-th roots of unity. But all those roots except 1 lie outside of $\mathbb{Q}$, since $p$ is odd. Therefore, any solution to $X^{p}+Y^{p}+Z^{p}=0$ must be of the form $(x: y:-1)$ or a permutation of this, which translates to an affine solution $(\lambda x, \lambda y,-\lambda)$ or a permutation. For our purposes the affine solution must end with -1 , in order to yield $X^{p}+Y^{p}=1$. Clearly $(x, y,-1)$ works, and choosing $\lambda=-\frac{1}{x}$ gives $\left(-1,-\frac{y}{x}, \frac{1}{x}\right)$, while choosing $\lambda=-\frac{1}{y}$ gives $\left(-\frac{x}{y},-1, \frac{1}{y}\right)$, which both can be permuted under $S_{3}$ to the appropriate form. However, no other value for $\lambda$ can give the necessary coordinate -1 .

Corollary 4.6. The six nontrivial solutions of $X^{p}+Y^{p}=1$ in $\mathbb{Q}\left(D_{p}\right)$ are $(x, y),\left(-\frac{y}{x}, \frac{1}{x}\right),\left(-\frac{x}{y}, \frac{1}{y}\right)$, and the transpositions of these, where $(x, y)$ is any one nontrivial solution.

So in Theorem 3.4, when we built the computable isomorphism $f$ from $F$ onto an arbitrary computable copy $\tilde{F}$, there were actually six possible images for each $x_{s}$ in $\tilde{F}$, which we identified by finding nontrivial solutions to $d_{p_{s}}=0$. We simply chose $f\left(x_{s}\right)$ to be the first one we recognized, since Theorem 2.8 shows that each of the six is a valid choice. For Theorem 4.2, however, we need a transcendence basis each element of which has only one possible image in the target field.

Proof. We now prove Theorem 4.2. Let $F$ be the computable field defined in Theorem 3.4, presented as

$$
F=\mathbb{Q}\left(x_{0}, x_{1}, \ldots\right)\left[y_{0}, y_{1}, \ldots\right] /\left(d_{p_{i}}\left(x_{i}, y_{i}\right): i \in \omega\right),
$$

where $\left\langle p_{i}\right\rangle_{i \in \omega}$ was the computable sequence of primes chosen there. In the nice presentation $F$ constructed there, the transcendence basis $B=\left\{x_{i}: i \in \omega\right\}$ was computable, of course, but not intrinsically computable, as noted above. However, we can build an intrinsically computable basis $A$ from it. 
The nontrivial solutions of each $d_{p_{i}}(X, Y)=0$ in $F$ were given by Proposition 4.5: $\left\langle x_{i}, y_{i}\right\rangle,\left\langle y_{i}, x_{i}\right\rangle,\left\langle\frac{1}{y_{i}}, \frac{-x_{i}}{y_{i}}\right\rangle$, $\left\langle\frac{-x_{i}}{y_{i}}, \frac{1}{y_{i}}\right\rangle,\left\langle\frac{1}{x_{i}}, \frac{-y_{i}}{x_{i}}\right\rangle,\left\langle\frac{-y_{i}}{x_{i}}, \frac{1}{x_{i}}\right\rangle$, and no others. For each $i$, let $z_{i}$ be the sum of these six numbers:

$$
z_{i}=x_{i}+y_{i}+\frac{1}{y_{i}}-\frac{x_{i}}{y_{i}}+\frac{1}{x_{i}}-\frac{y_{i}}{x_{i}}=\left(\frac{1-x_{i}}{1-x_{i}^{p_{i}}}\right) y_{i}^{p_{i}-1}+\left(\frac{x_{i}-1}{x_{i}}\right) y_{i}+\frac{x_{i}^{2}+1}{x_{i}},
$$

where we have used the algebraic relation $y_{i}^{p_{i}}=1-x_{i}^{p_{i}}$ to rewrite the expression as a polynomial in $y_{i}$ over $\mathbb{Q}\left(x_{i}\right)$. Thus $z_{i}$ clearly lies in $\mathbb{Q}\left(x_{i}\right)\left[y_{i}\right]$ but not in $\mathbb{Q}$, as $y_{i}$ is algebraic of degree $p_{i}$ over $\mathbb{Q}\left(x_{i}\right)$, and so $z_{i}$ is algebraically interdependent with $x_{i}$ in $F$ over $\mathbb{Q}$. So we may replace each $x_{i}$ by $z_{i}$ in the basis $B$, giving a new transcendence basis $A=\left\{z_{i}: i \in \omega\right\}$ for $F$.

Now each $z_{i}$ is defined by a simple existential formula $\psi_{i}(z)$ : it is the sum of six distinct elements $x \in F$, each satisfying

$$
x \neq 0 \& x \neq 1 \&(\exists y \in F)\left[x^{p_{i}}+y^{p_{i}}=1\right] .
$$

By Proposition 4.5, this formula $\psi_{i}(z)$ actually defines $z_{i}$ in $F$. So the transcendence basis $A$ is defined by

$$
(\exists i \in \omega) \psi_{i}(z)
$$

(This definition is in fact a computable infinitary $\Sigma_{1}$ formula, quantifying over $\omega$ as well as over $E$, which is acceptable for us, though less common in model theory.) Therefore, the image $f(A)$ under any isomorphism $f$ (not necessarily computable) from $F$ onto any computable field $\tilde{F}$ must also be existentially defined in $\tilde{F}$, hence c.e. Now we invoke a simple lemma to show that $f(A)$ is computable in $\tilde{F}$.

Lemma 4.7. In a computable field $K$, if a transcendence basis $A$ is computably enumerable, then $A$ is computable.

Proof. $K$ is algebraic over the purely transcendental extension $Q(A)$, where $Q$ is the prime subfield of $K$. Given any $t \in K$, we find an $n \in \omega$ and a nonzero polynomial $p(T) \in Q\left(a_{0}, \ldots, a_{n}\right)[T]$ with $p(t)=0$, by enumerating $A=\left\{a_{0}, a_{1}, \ldots\right\}$ and searching though such polynomials (for all $n$ simultaneously). But then $t$ is algebraic over $Q\left(a_{0}, \ldots, a_{n}\right)$, and so $t \in A$ if and only if $t \in\left\{a_{0}, \ldots, a_{n}\right\}$.

This completes the proof of Theorem 4.2.

We remark that Proposition 4.5 was essential to this proof of Theorem 4.2. In a field as described more generally in Theorem 3.5, one might not be able to compute the number of solutions to $C_{i}$ in its function field, and therefore it could be impossible to state the definitions $\psi_{i}(z)$ uniformly in $i$.

We also remark that this same proof shows the basis $A$ to be relatively intrinsically computable: its image $f(A)$ under any isomorphism $f$ is computable relative to the Turing degree of the field $f(F)$. Likewise, this field $F$, and also those described by Theorem 3.5, are relatively computably categorical: if $\tilde{F}$ is a field isomorphic to $F$ but of arbitrary Turing degree, then there is an isomorphism between these fields which is computable in the degree of $\tilde{F}$.

\section{Questions}

Several basic questions arise from the algebraic geometry presented here. For instance, we do not know whether cover relations exist among any of the Fermat curves $D_{p}$ and $D_{q}$ with $3<p<q . D_{3}$ is a special case, of course, since its genus equals 1 , and in Theorem 3.1 we chose specific primes such that no cover relations exist among those curves. But the general case remains unclear.

Also, while Theorems 3.4 and 3.5 give examples of computably categorical fields of infinite transcendence degree, they leave open the larger question of determining a structural criterion for computable categoricity of fields. If anything, they make this question appear more difficult, since now we know that infinite transcendence degree is not sufficient to rule out computable categoricity. 
Specifically, we remarked in Lemma 2.7 that there is no cover relation among any collection of curves of any single fixed genus $\geq 2$. Whether this gives rise to other computably categorical fields of infinite transcendence degree depends on whether one can produce a computable collection of such curves with the effective Faltings property. It seems plausible that this can be done, but the proof remains elusive, and introduces the larger question of computing the number of solutions in $\mathbb{Q}$ for an arbitrary collection of curves with the (not necessarily effective) Faltings property. Of course, the exact number of $\mathbb{Q}$-rational points on a curve given by a polynomial $q \in \mathbb{Q}[X, Y]$ defines a limitwise monotonic function $\lim _{s} g(q, s)$ (that is, with $g$ computable and with $g(q, s) \leq g(q, s+1)$ for all $q$ and $s$ ), and when we restrict to curves of genus $\geq 2$, this function is total, i.e., the limit is always finite. However, it remains unknown whether the exact number is computable from $q$ or not.

We regard this as a natural and challenging question. It seems related to Hilbert's Tenth Problem for $\mathbb{Q}$, which demands a decision procedure for the existence of $\mathbb{Q}$-rational solutions to polynomials in arbitrarily many variables over $\mathbb{Q}$. In particular, an oracle for Hilbert's Tenth Problem for $\mathbb{Q}$ would allow one to determine the exact number of rational solutions to any curve of genus $\geq 2$ : one can express the question "does this curve have a solution distinct from the finitely many I have already found" in a uniform way such that the oracle could answer that question. This would not necessarily work for curves of genus $<2$, or for varieties in general, which could contain infinitely many rational points. (To decide which varieties have the Faltings property appears to require the jump of the oracle set for Hilbert's Tenth Problem for $\mathbb{Q}$.) So, even if the effective Faltings property holds for all curves of genus $\geq 2$, we would still not have an obvious algorithm for Hilbert's Tenth Problem for polynomials defining varieties of higher dimension.

\section{Acknowledgements}

Russell Miller was partially supported by NSF grant \# DMS - 1001306, by several grants from The City University of New York PSC-CUNY Research Award Program and the Queens College Research Enhancement Fund, and by grant \# 13397 from the Templeton Foundation. Both authors were supported by CUNY Collaborative Grant \# 80209-04-12. Hans Schoutens was partially supported by NSF grant \# DMS-0500835, and by grants numbered 67195-00 36, 68093-00 37, 69135-00 38, and 61085-00 39 from The City University of New York PSC-CUNY Research Award Program.

The authors wish to acknowledge useful conversations with Gunther Cornelissen, Oleg Kudinov, Alexandra Shlapentokh, and Lucien Szpiro.

\section{References}

[1] M.H. Baker, E. González-Jiménez, J. González \& B. Poonen; Finiteness results for modular curves of genus at least 2. Amer. J. Math. 127 (2005) 6, 1325-1387.

[2] W. Calvert; The isomorphism problem for classes of computable fields, Archive for Mathematical Logic $\mathbf{4 3}$ (2004), 327-336.

[3] J. Cassels \& E. Flynn; Prolegomena to a middlebrow arithmetic of curves of genus 2, London Mathematical Society Lecture Note Series, vol. 230. (Cambridge: Cambridge University Press, 1996.)

[4] R.G. Downey, D.R. Hirschfeldt, \& B. Khoussainov; Uniformity in Computable Structure Theory, Algebra and Logic 42 (2003), 318-332.

[5] Yu.L. Ershov; Theorie der Numerierungen, Zeits. Math. Logik Grund. Math. 23 (1977), 289-371.

[6] Yu.L. Ershov \& S.S. Goncharov; Constructive fields, Section 2.5 in Constructive Models (New York: Kluwer Academic/Plenum Press, 2000).

[7] M.D. Fried \& M. Jarden; Field Arithmetic (Berlin: Springer-Verlag, 1986).

[8] A. Frohlich \& J.C. Shepherdson; Effective procedures in field theory, Phil. Trans. Royal Soc. London, Series A 248 (1956) 950, 407-432. 
[9] S.S. Goncharov; Autostability and computable families of constructivizations, Algebra and Logic 14 (1975), 647-680 (Russian), 392-409 (English translation).

[10] S.S. Goncharov; Nonequivalent constructivizations, Proc. Math. Inst. Sib. Branch Acad. Sci. (Novosibirsk: Nauka, 1982).

[11] S.S. Goncharov; Autostable models and algorithmic dimensions, Handbook of Recursive Mathematics, vol. 1 (Amsterdam: Elsevier, 1998), 261-287.

[12] S.S. Goncharov \& V.D. Dzgoev; Autostability of models, Algebra and Logic 19 (1980), 45-58 (Russian), 28-37 (English translation).

[13] S.S. Goncharov, S. Lempp \& R. Solomon; The computable dimension of ordered abelian groups, Advances in Mathematics 175 (2003) 1, 102-143.

[14] A. Grothendieck; Revêtements étales et groupe fondamental (SGA 1), Séminaire de géométrie algébrique du Bois Marie 1960-61, Documents Mathématiques (Paris) 3, Société Mathématique de France, Paris, 2003. Updated and annotated reprint of the original [Berlin: Springer, Lecture Notes in Mathematics 224, 1971].

[15] V.S. Harizanov; Pure computable model theory, Handbook of Recursive Mathematics, vol. 1 (Amsterdam: Elsevier, 1998), 3-114.

[16] R. Hartshorne; Algebraic Geometry (New York: Springer-Verlag, 1977).

[17] D. Hirschfeldt, K. Kramer, R.G. Miller, \& A. Shlapentokh; Categoricity Properties for Computable Algebraic Fields, submitted for publication.

[18] G. Kempf; Algebraic Varieties, London Mathematical Society Lecture Note Series, vol. 172. (Cambridge: Cambridge University Press, 1993).

[19] B. Khoussainov \& R.A. Shore; Effective Model Theory: The Number of Models and their Complexity, Models And Computability: Invited Papers from Logic Colloquium '97, ed. S.B. Cooper \& J.K. Truss, LMSLNS 259 (Cambridge: Cambridge University Press, 1999), 193-240.

[20] L. Kronecker; Grundzüge einer arithmetischen Theorie der algebraischen Größen, J. f. Math. 92 (1882), 1-122.

[21] S. Lempp, C. McCoy, R. Miller, \& R. Solomon; Computable categoricity of trees of finite height, Journal of Symbolic Logic 70 (2005), 151-215.

[22] H.-W. Leopoldt; Über die Automorphismengrupper des Fermatkorpers, Journal of Number Theory 56 (1996) 2, 256-282.

[23] G. Metakides \& A. Nerode; Effective content of field theory, Annals of Mathematical Logic 17 (1979), 289-320.

[24] R.G. Miller; The computable dimension of trees of infinite height, Journal of Symbolic Logic 70 (2005), 111-141.

[25] R.G. Miller; Computable fields and Galois theory, Notices of the AMS 55 (August 2008) 7, $798-807$.

[26] R.G. Miller; Computability and differential fields: a tutorial, to appear in Differential Algebra and Related Topics: Proceedings of the Second International Workshop, eds. L. Guo \& W. Sit (World Scientific). Also available at qcpages. qc. cuny. edu/ rmiller/research.html.

[27] R.G. Miller; $\boldsymbol{d}$-Computable categoricity for algebraic fields, Journal of Symbolic Logic 74 (2009) 4, 13251351.

[28] R.G. Miller, \& A. Shlapentokh; Computable categoricity for algebraic fields with splitting algorithms, submitted for publication.

[29] B. Poonen; Unramified covers of Galois covers of low genus curves, Math Res. Lett. 12 (2005) 4, 475-481.

[30] M. Rabin; Computable algebra, general theory, and theory of computable fields, Transactions of the American Mathematical Society 95 (1960), 341-360. 
[31] J.B. Remmel; Recursively categorical linear orderings, Proceedings of the American Mathematical Society 83 (1981), 387-391.

[32] J.B. Remmel; Recursive isomorphism types of recursive Boolean algebras, Journal of Symbolic Logic 46 (1981), 572-594.

[33] R.I. Soare; Recursively Enumerable Sets and Degrees (New York: Springer-Verlag, 1987).

[34] V. Stoltenberg-Hansen \& J.V. Tucker; Computable Rings and Fields, in Handbook of Computability Theory, ed. E.R. Griffor (Amsterdam: Elsevier, 1999), 363-447.

[35] P. Tzermias; The group of automorphisms of the Fermat curve, Journal of Number Theory 53 (1995) 1 , $173-178$. 